
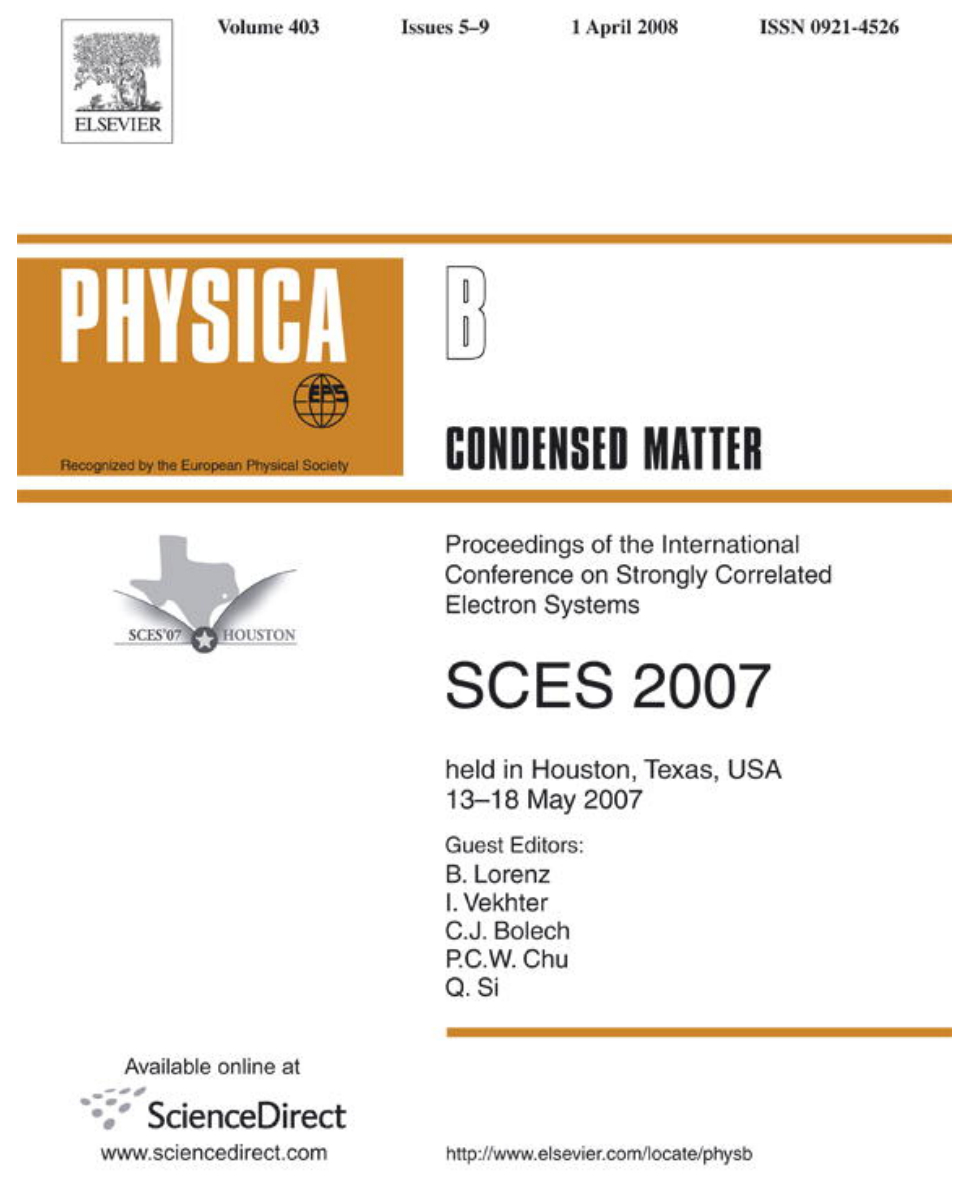

This article was published in an Elsevier journal. The attached copy

is furnished to the author for non-commercial research and education use, including for instruction at the author's institution, sharing with colleagues and providing to institution administration.

Other uses, including reproduction and distribution, or selling or licensing copies, or posting to personal, institutional or third party websites are prohibited.

In most cases authors are permitted to post their version of the article (e.g. in Word or Tex form) to their personal website or institutional repository. Authors requiring further information regarding Elsevier's archiving and manuscript policies are encouraged to visit:

http://www.elsevier.com/copyright 


\title{
Vibrational effects on low-temperature properties of molecular conductors
}

\author{
Jernej Mravlje ${ }^{\mathrm{a}, *}$, Anton Ramšak ${ }^{\mathrm{b}, \mathrm{a}}$, Rok Žitko ${ }^{\mathrm{a}}$ \\ ${ }^{a}$ Jožef Stefan Institute, Jamova 39, Si-1000 Ljubljana, Slovenija \\ ${ }^{\mathrm{b}}$ Faculty of Mathematics and Physics, University of Ljubljana, Jadranska 19, Si-1000 Ljubljana, Slovenija
}

\begin{abstract}
We calculate characteristic correlation functions for the Anderson model with additional phonon-assisted coupling to the odd conduction channel. This model describes, for example, the behavior of a molecule embedded between two electrodes in linear transport experiments where the position of the molecule with respect to the leads affects the tunneling amplitudes. We use variational projectionoperator method and numerical renormalization group (NRG) method. The spin is Kondo screened either by even or odd conduction channel depending on the gate voltage and electron-phonon coupling. However, in all regimes the gate-voltage dependence of the zero temperature conductance is found to be qualitatively the same as in the model with no coupling to the vibrational mode.
\end{abstract}

(C) 2007 Elsevier B.V. All rights reserved.

PACS: 72.15.Qm; 73.23.-b; 73.22.-f

Keywords: Kondo effect; Molecular conductors; Dynamic symmetry breaking

In recent years the studies of quantum impurity systems have undergone a considerable revival due to improvements in experimental techniques for measuring the electron transport through quantum dots and single molecules, as well as due to the development of the DMFT technique which maps interacting lattice problems to quantum impurity problems with an additional selfconsistency condition. The prototype model for this class of problems is the Anderson model for a single impurity in a metallic host with $H_{\text {imp }}=U n_{\uparrow} n_{\downarrow}+\varepsilon n$, where $n=n_{\uparrow}+n_{\downarrow}$ is the number of electrons occupying the impurity orbital with energy $\varepsilon$ relative to the chemical potential, and with the Coulomb repulsion $U$ due to the double occupancy of the impurity orbital.

An important class of quantum impurity models include coupling to the bosonic degrees of freedom describing the vibrational modes of the molecule or phonons in the bulk. There are two basic types of the electron-phonon coupling, (i) the Holstein coupling of form $n x$, where $n$ is the electron

\footnotetext{
${ }^{*}$ Corresponding author. Fax: +386614773724

E-mail address: jernej.mravlje@ijs.si (J. Mravlje).
}

density and $x$ the oscillator displacement, and (ii) the coupling to hopping term of form $v x$, where $v$ is the hopping (hybridization) operator that couples the impurity to the conduction band. While type (i) is more relevant when the oscillation is related to the change in volume to which the electron is confined (breathing modes), type (ii) is relevant whenever the displacement modulates the hopping probability.

The addition of the Holstein term to the Anderson Hamiltonian effectively reduces the Coulomb repulsion and the hybridization $\Gamma$. The effect of the electrons on the phonon propagator is also interesting: when effective $U$ changes sign, a peak in the phonon propagator at reduced frequencies (the 'soft mode') emerges [1,2]. The soft mode is related to the charge susceptibility, which is increased in this regime [3].

Very recently, similar behavior was found also in the case where the electron-phonon coupling term is of the form $H_{\mathrm{el}-\mathrm{ph}}=g x v_{\mathrm{odd}}$, where $v_{\text {odd }}$ describes the hopping from impurity orbital to the odd conduction channel (antisymmetric combination of the orbitals of the noninteracting part of the Hamiltonian) [4]. The model without 
phonons consist of $v_{\text {even, }}$ which couples the impurity only to the even conduction channel (symmetric combination of orbitals of the noninteracting part of Hamiltonian).

The same model (but for finite $U$ instead of $U \rightarrow \infty$ treatment of Ref. [4]) was analyzed also with the variational projection-operator method [5]. In this method the ground state is expressed in terms of the ground state of an auxiliary noninteracting Hamiltonian [6]. Several variants were tested, with parameters chosen so as to allow for coupling, (i) only to even channel, (ii) only to odd channel, (iii) a combination of both. Variational method applied to variant (iii) leads in certain parameter regimes to a ground state of broken parity symmetry (see Fig. 1(a,b)), marked by non-vanishing expectation value of displacement (Fig. 1(c), thin-dotted) and consequently [5] considerably reduced conductance through a molecule (plotted as a function of departure from particle-hole symmetric point $\delta=\varepsilon+U / 2$ in Fig. 1(d)). As discussed below, the ground state should have a well defined parity, therefore only the variants (i) and (ii) of the variational procedure correspond to the ground state of correct symmetry. While it would be instructive to implement the variational method in a manner which correctly took into account the tunneling between the classically degenerate minima of the oscillator potential [4], it currently appears that the implementation would require calculating matrix elements between two distinct Hartree-Fock vacua, which precludes the use of Wick's theorem upon which our current implementation of the variational procedure is based [6].

We thus present preliminary results obtained with the NRG method, which does not suffer from this problem. The parity in NRG results is not broken; the expectation a

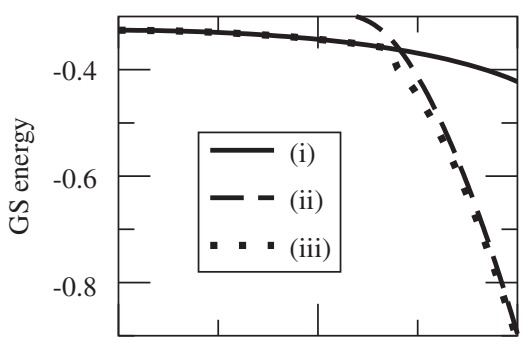

C

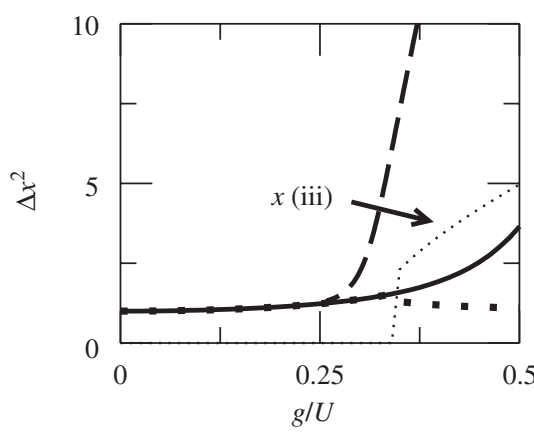

b

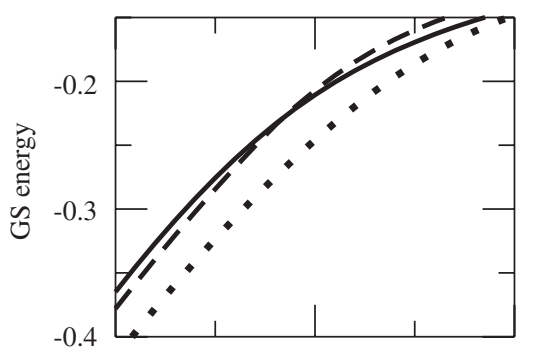

d

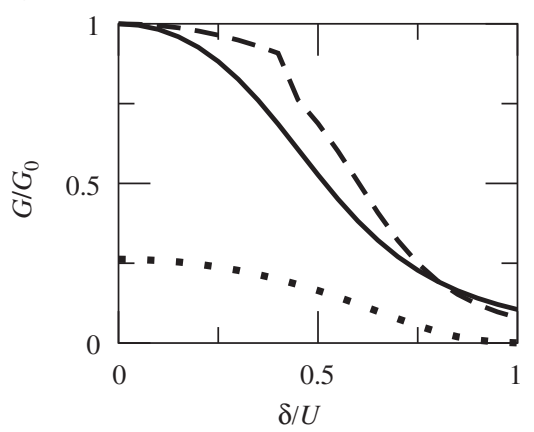

Fig. 1. Variational results $[U / \Gamma=5, \Gamma / D=0.04, \Omega=2.5 \Gamma ; \Omega$ is the phonon frequency, $D$ the bandwidth. $\delta=0$ (left pannels), $g / U=0.36$ (right pannels)]: (a, b) Variational ground state energy. (c) Displacement fluctuations and displacement for (iii). In (i) and (ii) the parity symmetry is retained, hence the displacement vanishes. (d) The conductance.

a

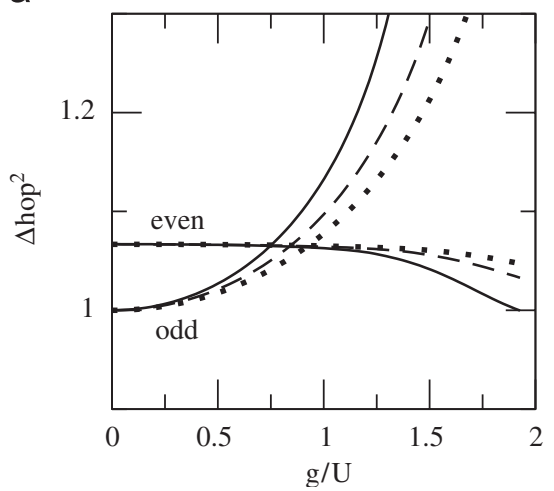

b

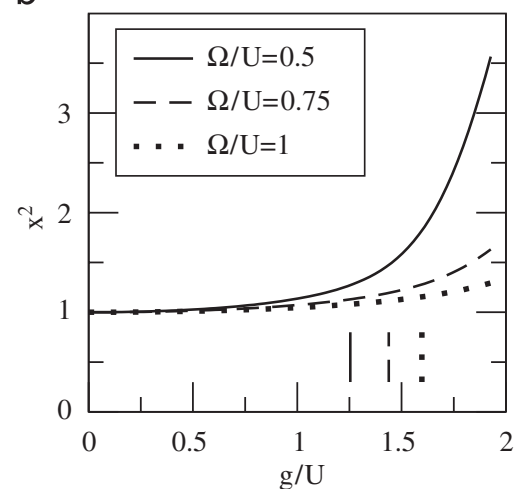

Fig. 2. NRG results $[U / \Gamma=25, D=U]$ : (a) fluctuations of hopping to even (full) and odd (dashed) channel, (b) fluctuations of displacement. 


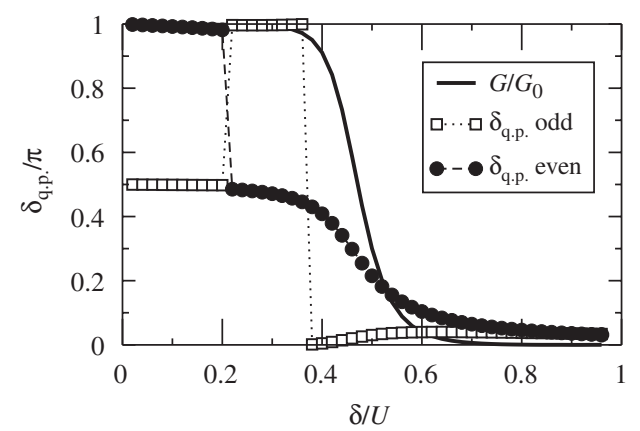

Fig. 3. NRG results $[U / \Gamma=25, D=U, \Omega / U=1, g / \Omega=1.72]$ : Zero temperature quasiparticle scattering phase shifts in even (circles) and odd (squares) channel plotted versus departure from $\mathrm{p}-\mathrm{h}$ symmetry calculated using NRG. Corresponding conductance is also plotted (dashed). Note that the phase shifts are defined modulo $\pi$.

values of the displacement and of the hopping term $v_{\text {odd }}$ thus vanish. In Fig. 2(a) we compare the fluctuations of hopping to even and odd channels as a measure of the 'activity' of corresponding channels. For $g$ large enough the latter are larger, corresponding to increased fluctuations of the displacement and the emergence of the soft mode. The ground state of the system, as seen from the NRG renormalization flow (not shown here) corresponds to the Fermi liquid ground state of the single-channel Kondo problem [7] with the characteristic quasi-particle scattering phase shift $\delta_{\text {q.p. }} \sim \pi / 2$ in the even or odd channel, depending on whether the effective phonon mediated coupling to the odd channel is smaller or larger than the direct coupling to the even channel. When the couplings match (marked in Fig. 2(b) by vertical lines), an unstable fixed-point of the two-channel Kondo model type is found.

In Fig. 3 the scattering phase-shifts are plotted as a function of $\delta$. The coupling to the phonon-assisted channel is chosen so that in the ground state the impurity spin is screened by the odd channel for small $\delta$, while for larger $\delta$ it is screened by the even channel, as seen from $\delta_{\text {q.p.; even,odd }} \sim \pi / 2$, respectively. Further away from the symmetric point the model is tuned into the valence fluctuating regime where the Kondo effect is suppressed. When the model is used to describe a molecule (or a quantum dot) embedded between two leads, the scattering phase shifts directly determine the differential conductance (Fig. 3 dashed). The conductance curve shown is qualitatively equal to that of the generic one-electron transistor in the single-channel Kondo regime, despite the fact that the Kondo effects occurs in different channels as the gate voltage is swept.

The work was supported by SRA under grant P1-0044.

\section{References}

[1] A.C. Hewson, D. Meyer, J. Phys.: Condens. Matter 14 (2002) 427.

[2] G.S. Jeon, T. Park, H. Choi, Phys. Rev. B 68 (2003) 045106.

[3] J. Mravlje, A. Ramšak, T. Rejec, Phys. Rev. B 72 (2005) 121403(R).

[4] C.A. Balseiro, P.S. Cornaglia, D.R. Grempel, Phys. Rev. B 74 (2006) 235409.

[5] J. Mravlje, A. Ramšak, T. Rejec, Phys. Rev. B 74 (2006) 205320.

[6] T. Rejec, A. Ramšak, Phys. Rev. B 68 (2003) 033306.

[7] For a review of the Kondo physics see: A.C. Hewson, The Kondo Problem to Heavy Fermions, University Press, Cambridge, 1993. 